\title{
ESTIMATIVAS DE EVAPOTRANSPIRAÇÃO E AS CONSEQÜÊNCIAS DEVIDO AOS ERROS NA DETERMINAÇÃO DE SALDO DE RADIAÇÃO E EFEITOS ADVECTIVOS
}

\author{
Gertrudes Macario de Oliveira ${ }^{1}$ \& Mário de Miranda Vilas Boas Ramos Leitão ${ }^{2}$
}

\section{RESUMO}

\begin{abstract}
Objetivou-se com esse estudo, analisar as conseqüências nas estimativas da evapotranspiração (ET) durante o ciclo de desenvolvimento de uma cultura de amendoim, devido aos erros cometidos na determinação do saldo de radiação $(\mathrm{Rn})$ e àqueles causados pelos efeitos advectivos. Esta pesquisa foi conduzida no perímetro irrigado da Estação Experimental da CODEVASF, na cidade de Rodelas, BA, no período de setembro a dezembro de 1996. Os resultados mostraram que erros no cálculo de Rn de até $2,2 \mathrm{MJ} \mathrm{m}^{-2} \mathrm{~d}^{-1} \mathrm{e}$, conseqüentemente, na estimativa da ET, podem ser cometidos dependendo do tempo considerado na totalização diária de Rn. Verificou-se, ainda, que tanto as áreas circunvizinhas próximas ao campo experimental, como as áreas de solos expostos entre fileiras dentro do próprio campo, contribuíram de forma significativa para a geração de advecção local de calor sensível e, portanto, para o aumento da evapotranspiração.
\end{abstract}

Palavras-chave: saldo de radiação, advecção, evapotranspiração

\section{EVAPOTRANSPIRATION ESTIMATES AND CONSEQUENCES DUE TO ERRORS IN THE DETERMINATION OF THE NET RADIATION AND ADVECTIVE EFFECTS}

\begin{abstract}
The objective of this study was to analyze the consequences in the evapotranspiration estimates (ET) during the growing cycle of a peanut crop due to the errors commited in the determination of the radiation balance ( $\mathrm{Rn})$, as well as those caused by the advective effects. This research was conducted at the Experimental Station of CODEVASF in an irrigated perimeter located in the city of Rodelas, BA, during the period of September to December of 1996. The results showed that errors of the order of $2.2 \mathrm{MJ} \mathrm{m}^{-2} \mathrm{~d}^{-1}$ in the calculation of Rn, and consequently in the estimate of ET, can occur depending on the time considered for the daily total of Rn. It was verified that the surrounding areas of the experimental field, as well as the areas of exposed soil within the field, contributed significantly to the generation of local advection of sensible heat, which resulted in the increase of the evapotranspiration.
\end{abstract}

Key words: net radiation, advection, evapotranspiration

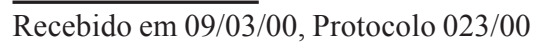

${ }^{1}$ Doutoranda do Curso de Recursos Naturais - CCT/UFPB, CEP 58109 - 970, Campina Grande, PB. Telefax: (0xx83) 3101202. E-mail gertrude@dca.ufpb.br

${ }^{2}$ Prof. Dr. em Meteorologia do Departamento de Ciências Atmosféricas - CCT/UFPB, CP 10099, CEP 58109 - 970, Campina Grande, PB. Telefax: (0xx83) 310 1202, Ramal: 32. E-mail miranda@dca.ufpb.br 


\section{INTRODUÇÃO}

O balanço de radiação à superfície representa a contabilidade dos fluxos radiativos à superfície, ou seja, é a soma dos balanços de radiação de ondas curtas e de ondas longas; portanto, seu conhecimento é fundamental para melhor planejamento das atividades agrícolas e, dentro deste contexto, o saldo de radiação representa a principal fonte de energia utilizada nos diversos processos físico-químicos que ocorrem na superfície e o principal parâmetro utilizado em muitos métodos que estimam as perdas de água de superfícies vegetadas para a atmosfera. Portanto, para obter-se estimativas corretas de evapotranspiração, é muito importante ter-se o saldo de radiação medido ou estimado de forma correta e precisa. Dentre os estudos envolvendo o saldo de radiação, destacam-se os de: Linacre (1968), Villa Nova (1973) e Leitão (1994) entre outros.

Por outro lado, também é fundamental considerar-se, nas estimativas de evapotranspiração, a energia adicional na forma de advecção, visto que a advecção de calor sensível de superfícies descobertas e secas situadas nas vizinhanças da área em questão, pode constituir-se uma fonte significativa de energia para aumentar as perdas de água por evapotranspiração, especialmente em regiões áridas e semi-áridas (Monteny, 1972; Rosenberg, 1972; Villa Nova, 1973; Leitão, 1989; Prueger et al., 1996). Ao se estudar a advecção de calor sensível sobre uma área vegetada, três situações podem identificar as fontes de origem do ar: adveção entre fileiras, causada pela exposição de solo dentro do dossel; advecção de bordadura ou local, originada por corrente de ar quente proveniente de superfícies com descontinuidade de temperatura, umidade e rugosidade; e advecção regional ou de larga-escala, massas de ar quente e seco originadas em grandes regiões quentes e secas, que se move através de uma superfície vegetada úmida (Hanks et al., 1971, Brakke et al., 1978).

Rosenberg \& Verma (1978) verificaram, ao conduzirem estudo em cultura de alfalfa irrigada em Mead - Nebraska, durante o período de seca prolongada em 1976, a existência de uma grande contribuição advectiva de calor sensível. A evapotranspiração atingiu valores de 4,75 a 14,22 $\mathrm{mm} \mathrm{d}^{-1}$, excedendo em $10 \mathrm{~mm} \mathrm{~d}^{-1}$ no terceiro dia estudado, quando $\mathrm{Rn}$ equivaleu a $7 \mathrm{~mm} \mathrm{~d}^{-1}$ e a ET durante todo período experimental, excedeu a soma $(\mathrm{Rn}+\mathrm{G})$. Por outro lado, Leitão et al. (1990) estudando o balanço de radiação e energia numa cultura de soja irrigada nas condições semi-áridas do Nordeste do Brasil, detectaram que $23 \%$ da energia utilizada na evapotranspiração foram fornecidos por advecção.

Chin Choy \& Kanemasu (1974) estudando os efeitos do espaçamento entre fileiras sobre o balanço de energia em cultura de sorgo, concluíram que o dossel de fileiras largas consumiu cerca de $10 \%$ a mais de água que o dossel de fileiras estreitas, com o calor sensível entre os dois espaçamento de fileiras, tendo contribuído significativamente para as diferenças em ET e favorecendo uma transpiração maior das plantas de fileiras largas; eles observaram, ainda, que as elevadas taxas de evapotranspiração sobre fileiras largas na primeira parte do período de crescimento foram provenientes da advecção entre fileira, enquanto no final deste período, resultaram da advecção de larga-escala, razão pelo qual os autores sugerem que a redução do calor sensível e, conseqüentemente, da evapotranspiração, pode ser obtida reduzindo-se o espaçamento entre fileiras.
Portanto, considerando-se que a evapotranspiração é freqüentemente comparada à energia disponível $(\mathrm{Rn})$ ou estimada com base nesta e que o seu conhecimento preciso e da advecção são de fundamental importância nos estudos das perdas de água por evapotranspiração em regiões semi-áridas, durante todo o ciclo de desenvolvimento das culturas, o presente estudo tem, como objetivo, mostrar que erros significativos nas estimativas da evapotranspiração podem ser cometidos e, em conseqüência levar a erros na reposição de água por irrigação, quando no cálculo de Rn não se considera o mesmo período contabilizado para a evapotranspiração ou, ainda, quando não se leva em conta os efeitos advectivos sobre a área cultivada.

\section{MATERIAL E MÉTODOS}

Este estudo foi conduzido no período de 20 de setembro a 23 de dezembro de 1996, no perímetro irrigado da Estação Experimental da Companhia de Desenvolvimento do Vale do São Francisco - CODEVASF, no município de Rodelas, BA (lat. 0850'S; long. 3846'W; alt. 270 m) utilizando-se o amendoim (Arachis hipogaea L.) cultivar BR-1. Para monitorar o experimento, três torres micrometeorológicas foram instaladas ao longo do campo experimental, na direção predominante do vento, distanciadas $25 \mathrm{~m}$ uma da outra (Figura 1) com sensores para medir temperatura do ar, e temperatura úmida a 1 e $2 \mathrm{~m}$, radiação solar incidente e refletida, saldo de radiação, fluxo de calor no solo e velocidade do vento a 1 e $2 \mathrm{~m}$. A aquisição dos dados foi efetuada através de um Micrologger 21X, a cada segundo, o que possibilitou médias a cada $5 \mathrm{~min}$, a partir das quais foram determinados os totais diários. Para efeito das análises, o campo experimental foi dividido em duas áreas: área 1, que compreendeu a primeira metade do campo (entre as torres 1 e 2) e área 2, como sendo a segunda metade do campo (entre as torres 2 e 3 ).

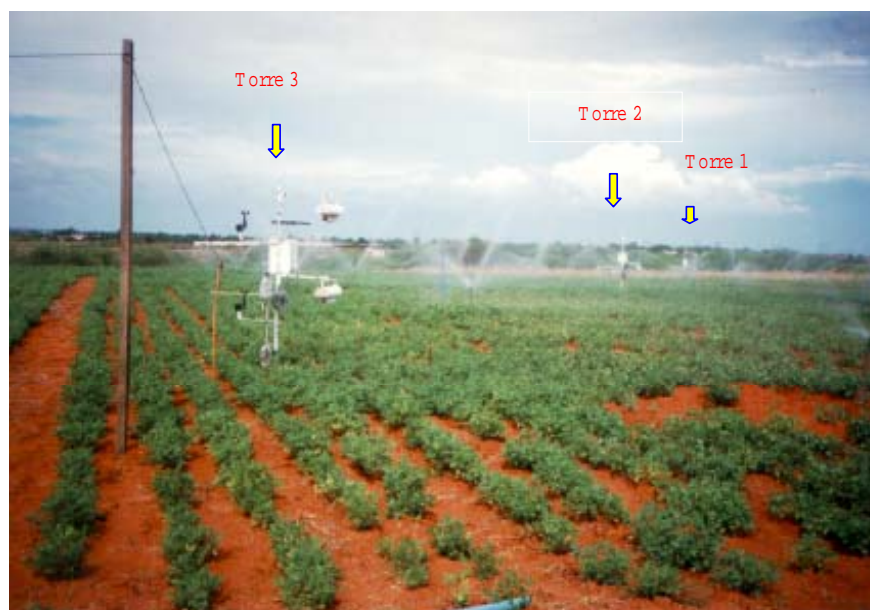

Figura 1. Posição das torres micrometeorológicas no campo experimental

\section{Balanço de radiação}

O balanço de radiação à superfície é dado pela soma dos fluxos de radiação de ondas curtas e ondas longas, e pode ser expresso pela seguinte equação:

$$
\mathrm{Rn}=(\mathrm{K} \downarrow-\mathrm{K} \uparrow)+(\mathrm{L} \downarrow-\mathrm{L} \uparrow)
$$


donde:

Rn - saldo de radiação

$\mathrm{K} \downarrow$ - radiação de ondas curtas incidente

$\mathrm{K} \uparrow$ - radiação de ondas curtas refletida pela superfície

$\mathrm{L} \downarrow \quad$ - radiação de ondas longas da atmosfera

$\mathrm{L} \uparrow \quad$ - radiação de ondas longas emitida pela superfície.

\section{Balanço de energia}

Em sua forma simplificada, o balanço de energia pode ser escrito como:

$$
\mathrm{Rn}=\mathrm{LE}+\mathrm{H}+\mathrm{G}+\mathrm{A}
$$

em que:

Rn - o saldo de radiação,

LE - fluxo de calor latente, sendo L o calor latente de evaporação $(\mathrm{L}=595-0,51 \mathrm{t})$, onde t é em ${ }^{\circ} \mathrm{C}$

H - fluxo de calor sensível,

G - fluxo de calor no solo, e

A - energia utilizada no processo fotossintético pelas plantas, a qual, segundo Villa Nova (1973) por ser muito pequena pode ser negligenciada.

Por definição, a razão entre o fluxo de calor sensível e o fluxo de calor latente (H/LE) é denominada razão de Bowen $(\beta)$; logo, substituindo-a na Eq. (2) tem-se:

$$
\mathrm{Rn}=\mathrm{LE}(1+\beta)+\mathrm{G}
$$

onde:

$$
\beta=\frac{c_{p} K_{h}}{L_{w}} \frac{\Delta T}{\Delta q}
$$

onde:

$\mathrm{c}_{\mathrm{p}}$ - calor específico do ar,

$\mathrm{K}_{\mathrm{h}}$ e $\mathrm{K}_{\mathrm{w}}$ - coeficiente de transferência turbulenta para o calor sensível e vapor d'água

$\Delta T / \Delta q$ - razão entre as diferenças de temperatura e umidade específica do ar nos níveis $Z_{1}$ e $Z_{2}$.

Para corrigir os efeitos de instabilidade atmosférica, tomou-se como base o número de Richardson para alturas próximas da superfície, dado por:

$$
\mathrm{Ri}=\frac{\mathrm{g}\left(\frac{\Delta \mathrm{T}}{\Delta \mathrm{Z}}\right)}{\mathrm{T}\left(\frac{\Delta \mathrm{u}}{\Delta \mathrm{Z}}\right)^{2}}
$$

onde:

$\mathrm{T}$ - temperatura do ar $(\mathrm{K})$

g - aceleração da gravidade $\left(\mathrm{m} \mathrm{s}^{-2}\right)$

$\Delta \mathrm{u} / \Delta \mathrm{Z}$ - gradiente vertical de velocidade do vento.

Para condições de estabilidade:

$$
\phi_{\mathrm{h}}=\phi_{\mathrm{w}}=\phi_{\mathrm{m}}=\left(1-5 \mathrm{R}_{\mathrm{i}}\right)^{-1}
$$

Para condições de instabilidade:

$$
\phi_{\mathrm{h}}=\phi_{\mathrm{w}}=\phi_{\mathrm{m}}^{2}=\left(1-16 \mathrm{R}_{\mathrm{i}}\right)^{-0,5}
$$

donde:

$\phi_{\mathrm{h}}, \phi_{\mathrm{w}}$ e $\phi_{\mathrm{m}}$ - representam às funções de instabilidade para transferência turbulenta de calor sensível, vapor d'água e quantidade de massa, respectivamente.

Como tanto para os casos mencionados como para condições de neutralidade os coeficientes de transferência turbulenta de calor sensível e vapor d'água são iguais $\left(\mathrm{K}_{\mathrm{h}}=\mathrm{K}_{\mathrm{w}}\right)$, substituindo-se a umidade específica $(\mathrm{q}=0,622 \mathrm{e} / \mathrm{P})$ na Eq. (4) tem-se:

$$
\beta=\frac{c_{p} P}{0,622 L} \frac{\Delta T}{\Delta e}
$$

em que:

$$
\begin{aligned}
& \gamma=c_{p} P / 0,622 \mathrm{~L} \text { - parâmetro psicrométrico } \\
& \mathrm{P} \quad \text { - pressão atmosférica }
\end{aligned}
$$

$\Delta \mathrm{T} / \Delta \mathrm{e}$ - razão entre as diferenças de temperatura e pressão de vapor, entre os níveis $Z_{1}$ e $Z_{2}$.

Logo:

$$
\beta=\gamma \frac{\Delta \mathrm{T}}{\Delta \mathrm{e}}
$$

Substituindo-se agora, a Eq. (9) na Eq. (3) tem-se:

$$
\mathrm{LE}=\frac{(\mathrm{Rn}-\mathrm{G})}{\left(1+\gamma \frac{\Delta \mathrm{T}}{\Delta \mathrm{e}}\right)}
$$

Para se obter a evapotranspiração, a Eq. (10) passa a ter a seguinte fórmula:

$$
\mathrm{ET}=\frac{(\mathrm{Rn}-\mathrm{G})}{\left(1+\gamma \frac{\Delta \mathrm{T}}{\Delta \mathrm{e}}\right) \mathrm{L}}
$$

Este método é conhecido como Método do Balanço de Energia. Conforme Verma et al. (1978) a suposição de igualdade entre os coeficientes de difusão turbulenta para o calor sensível e vapor d'água sob condições de advecção de calor sensível, pode causar erros significativos nas estimativas de LE efetuadas pelo método do balanço de energia. Para contornar o problema e corrigir esses erros, propuseram a seguinte expressão:

$$
\begin{gathered}
\frac{\mathrm{K}_{\mathrm{h}}}{\mathrm{K}_{\mathrm{w}}}=2,95+3,72 \frac{\Delta \mathrm{T}}{\Delta \mathrm{e}}+1,72\left(\frac{\Delta \mathrm{T}}{\Delta \mathrm{e}}\right)^{2} \\
-0,8>\frac{\Delta \mathrm{T}}{\Delta \mathrm{e}}>-0,1
\end{gathered}
$$


Deste modo, a razão de Bowen corrigida $\left(\beta_{\mathrm{c}}\right)$ passa a ter a seguinte forma:

$$
\beta_{\mathrm{c}}=\frac{\mathrm{Pc} \mathrm{c}_{\mathrm{p}}}{\mathrm{L} \varepsilon}\left[2,95+3,72 \frac{\Delta \mathrm{T}}{\Delta \mathrm{e}}+1,72\left(\frac{\Delta \mathrm{T}}{\Delta \mathrm{e}}\right)^{2}\right] \frac{\Delta \mathrm{T}}{\Delta \mathrm{e}}
$$

Portanto, a equação do balanço de energia à superfície pode ser escrita como:

$$
\mathrm{Rn}=\mathrm{LE}\left(1+\beta_{\mathrm{c}}\right)+\mathrm{G}
$$

Brakke et al. (1978) propuseram uma modificação no método do balanço de energia, incluindo a variação horizontal dos fluxos de calor latente e sensível. Desta forma, a divergência do fluxo de calor sensível entre a superfície e um nível acima, é dada por:

$$
\mathrm{H}_{1}-\mathrm{H}_{0}=\rho \mathrm{C}_{\mathrm{p}} \int_{0}^{\mathrm{z}_{1}} \mathrm{u} \frac{\partial \mathrm{T}}{\partial \mathrm{x}} \mathrm{dz}
$$

onde:

$\mathrm{H}_{1} \quad$ - o fluxo vertical de calor sensível para uma altura $Z_{1}$

$\rho \quad$ - a densidade do ar

$\mathrm{C}_{\mathrm{p}}$ - o calor específico do ar

u - a velocidade média do vento

$\Delta \mathrm{T} / \Delta \mathrm{x}$ - o gradiente horizontal de temperatura, na direção do vento predominante.

Similarmente, para o fluxo de calor latente, tem-se:

$$
\mathrm{LE}_{1}-\mathrm{LE}_{0}=\frac{\rho \mathrm{L} \varepsilon}{\mathrm{P}} \int_{0}^{\mathrm{z}_{1}} \mathrm{u} \frac{\partial \mathrm{e}}{\partial \mathrm{x}} \mathrm{dz}
$$

onde:

$$
\begin{aligned}
& \mathrm{P} \quad \text { - a pressão atmosférica } \\
& \varepsilon \quad \text { - a razão entre os pesos moleculares do vapor d'água } \\
& \quad \text { e ar seco }(\varepsilon=0,622) \\
& \text { e } \quad \text { - a pressão parcial do vapor d'água. }
\end{aligned}
$$

Tomando, agora, a equação do balanço de energia para um nível $\mathrm{z}_{\mathrm{o}}$ na forma que segue:

$$
\mathrm{LE}_{0}=\mathrm{Rn}-\mathrm{G}-\mathrm{H}_{0}
$$

Substituindo-se na Eq. (17) as Eq. (15) e (16) e considerando-se $\beta_{1}=\mathrm{H}_{1} / \mathrm{LE}_{1}$, tem-se:

$$
\mathrm{LE}_{0}=\left(\frac{\mathrm{Rn}-\mathrm{G}}{1+\beta_{1}}\right)-\left(\frac{\rho}{1+\beta_{1}}\right) \int_{0}^{\mathrm{z}_{1}} \mathrm{u}\left(\beta_{1} \frac{\mathrm{L} \varepsilon}{\mathrm{P}} \frac{\partial \mathrm{e}}{\partial \mathrm{x}}-\mathrm{c}_{\mathrm{p}} \frac{\partial \mathrm{T}}{\partial \mathrm{x}}\right) \mathrm{dz}
$$

Finalmente, substituindo-se na Eq. (18) a razão de Bowen $\left(\beta_{1}\right)$ pelo seu valor corrigido $\left(\beta_{c}\right)$ dado pela Eq. (13) tem-se:

$$
\mathrm{LE}_{0}=\left(\frac{\mathrm{Rn}-\mathrm{G}}{1+\beta_{\mathrm{c}}}\right)-\left(\frac{\rho}{1+\beta_{\mathrm{c}}}\right) \int_{0}^{\mathrm{z}_{1}} \mathrm{u}\left(\beta_{\mathrm{c}} \frac{\mathrm{L} \varepsilon}{\mathrm{P}} \frac{\partial \mathrm{e}}{\partial \mathrm{x}}-\mathrm{c}_{\mathrm{p}} \frac{\partial \mathrm{T}}{\partial \mathrm{x}}\right) \mathrm{dz}(19)
$$

A Eq. (19) representa a equação do método do balanço de energia acrescida do termo advectivo, a qual, ao ser dividida pelo calor latente de evaporação (L) resulta na seguinte expressão, para estimar a evapotranspiração corrigida dos efeitos advectivos:

$\mathrm{ET}=\left[\frac{\mathrm{Rn}-\mathrm{G}}{\left(1+\beta_{\mathrm{c}}\right) \mathrm{L}}\right]-\left[\frac{\rho}{\left(1+\beta_{\mathrm{c}}\right) \mathrm{L}}\right] \int_{0}^{\mathrm{z}_{1}} \mathrm{u}\left(\beta_{\mathrm{c}} \frac{\mathrm{L} \varepsilon}{\mathrm{P}} \frac{\partial \mathrm{e}}{\partial \mathrm{x}}-\mathrm{c}_{\mathrm{p}} \frac{\partial \mathrm{T}}{\partial \mathrm{x}}\right) \mathrm{dz}$

\section{RESULTADOS E DISCUSSÃO}

$\mathrm{Na}$ maior parte das atividades agrícolas que dependem de irrigação, a evapotranspiração (ET) é freqüentemente comparada à energia disponível (Rn) ou estimada com base nesta, porém quase sempre, enquanto a evapotranspiração é medida ou estimada entre $9 \mathrm{~h}$ de um dia e $9 \mathrm{~h}$ do dia seguinte, o saldo de radiação, em função de suas medidas serem efetuadas ao longo do dia, é calculado considerando-se o período do nascer ao pôr-do-sol, ou para $24 \mathrm{~h}$, ou seja, não se considera o mesmo intervalo de tempo usado na determinação da evapotranspiração. Conforme pode ser observado na Figura 2, quando se compara Rn calculado para o dia em questão com Rn calculado para o intervalo de tempo correspondente a $9 \mathrm{~h}$ do dia em questão às $9 \mathrm{~h}$ do dia seguinte, erros de até 2,2 $\mathrm{MJ} \mathrm{m}^{-2} \mathrm{~d}^{-1}$ podem ser cometidos. Deste modo, erros correspondentes serão cometidos nas estimativas de evapotranspiração e, como conseqüência, aplicações erradas de lâminas de água ao solo serão feitas por irrigação que, muitas vezes, podem representar água de mais ou de menos para a cultura. Tais erros podem ser ainda mais acentuados quando as irrigações são feitas com maior frequência e daí a importância de se ter medidas ou estimativas precisas da evapotranspiração.

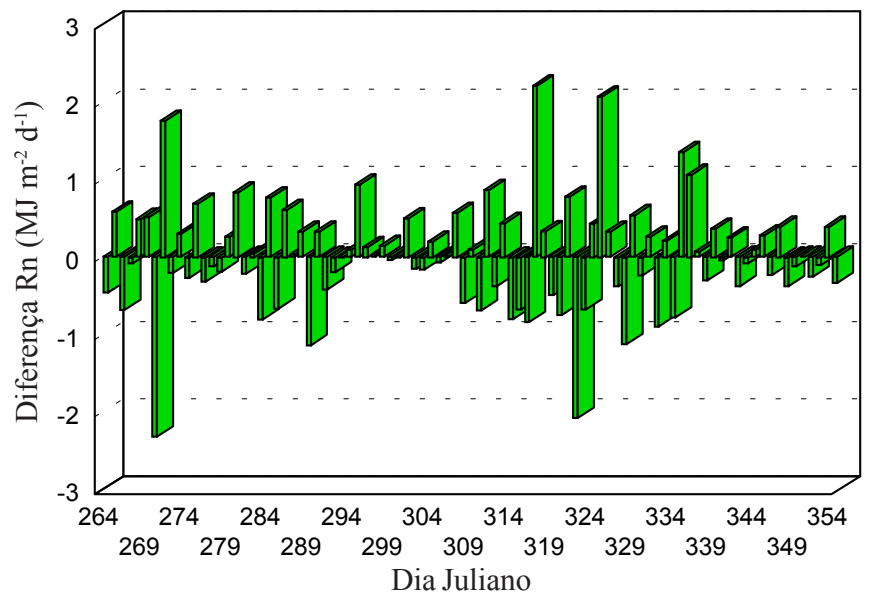

Figura 2. Diferença entre Rn integrado para o período diurno e integrado de $9 \mathrm{~h}$ do dia em questão, a $9 \mathrm{~h}$ do dia seguinte

Nas Figuras 3A e 3B são apresentados os valores diários integrados do fluxo de calor latente obtidos pelo método do balanço de energia original (LE) e o fluxo de calor latente corrigido dos efeitos advectivos (LEo) para todo o ciclo de desenvolvimento da cultura, nas duas áreas do campo experimental. Percebe-se que na área 1 (Fig. 3A) enquanto nas duas fases iniciais de desenvolvimento da cultura LEo $<\mathrm{LE}$, indicando ocorrência de advecção de ar úmido, devido às constantes irrigações numa área cultivada próxima, nas fases 
de floração e maturação verificou-se LEo > LE, indicando que houve advecção de calor sensível, ou seja, a medida em que a área vegetada próxima foi perdendo a folhagem e deixando de ser irrigada, passou a ter maior aquecimento do solo; com isto, o vento, ao passar sobre a mesma, atuou transportando ar quente e seco para a área 1 contribuindo, assim, para aumentar o fluxo de calor latente; já para a área 2 (Fig. 3B) percebe-se que praticamente durante todos os dias LEo $>$ LE, indicando que esta área foi influenciada pela entrada de ar quente e seco durante o ciclo de desenvolvimento da cultura, ou seja, desde a fase de solo descoberto até a fase de floração, a área 2 foi afetada por advecção local, gerada pelo aquecimento da superfície descoberta dentro da área experimental. $\mathrm{Na}$ fase de maturação ocorreu acentuada contribuição de energia adicional sobre a área 2 , que resultou da influência advectiva não apenas da área de solo descoberto entre fileiras dentro do próprio campo como, também, de áreas sem vegetação localizada ao sulsudeste do campo experimental.

(A) Área 1

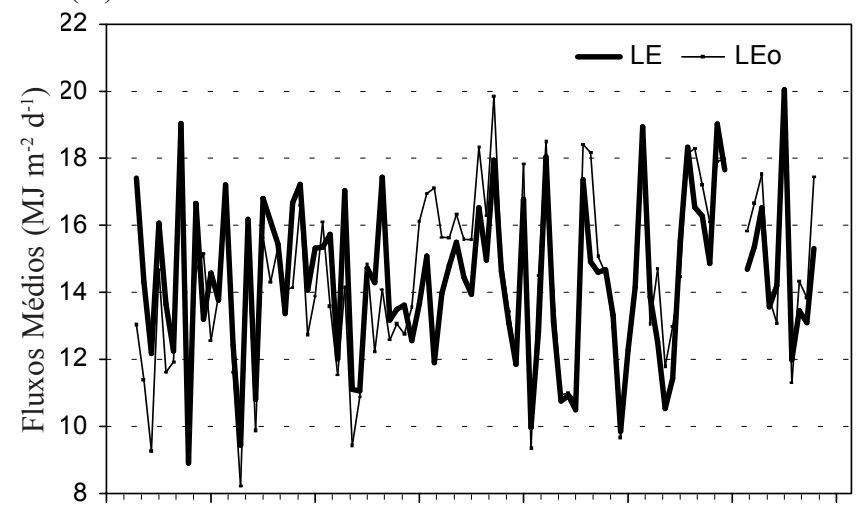

(B) Área 2

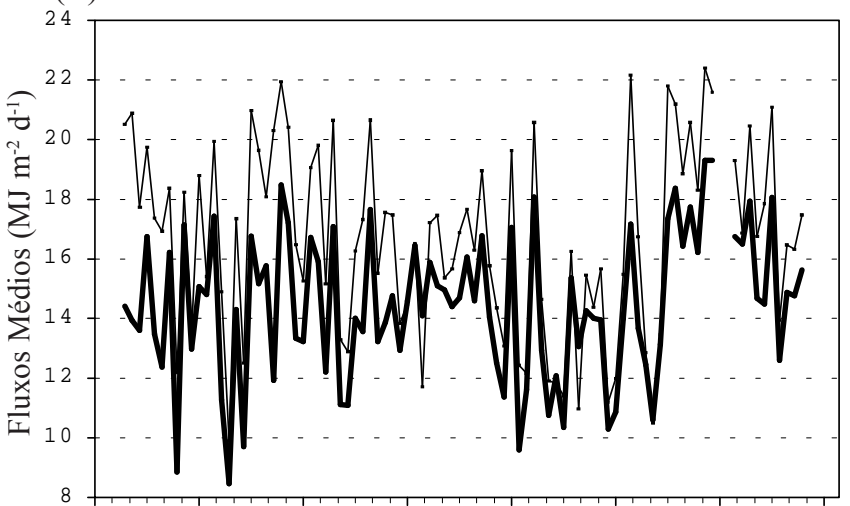

16-Set 30-Set 14-Out 28-Out 11-Nov 25-Nov 09-Dez 23-Dez Data

Figura 3. Fluxos LE e LEo obtidos pelo MBE original e corrigido da advecção, ao longo do ciclo de desenvolvimento da cultura: (A) área 1 e (B) área 2

\section{CONCLUSÕES}

Erros expressivos podem ser cometidos na estimativa da evapotranspiração (ET) quando Rn não é medido ou estimado corretamente para o mesmo período de tempo considerado, para ET, com isso diferenças de até $2,2 \mathrm{MJ} \mathrm{m}^{-2} \mathrm{~d}^{-1}$ podem ocorrer quando Rn é obtido diariamente, ao invés de $9 \mathrm{~h}$ de um dia a $9 \mathrm{~h}$ do dia seguinte. Procedimento utilizado quando se estima ET, visto que na pratica é inviável efetuar-se leituras de evapotranspirômetros e/ou tanques evaporimétricos diariamente à zero hora.

Percebeu-se também que, enquanto a primeira metade do campo experimental houve contribuição advectiva de ar úmido, da fase inicial ao início da floração na segunda metade do campo experimental houve advecção de ar seco, gerada pelo aquecimento do solo da área experimental, devido uma razoável porção deste ter permanecido descoberta durante o ciclo de desenvolvimento da cultura. Tais situações, caracterizam a ação da advecção de ar úmido entrando na primeira metade do campo experimental, que contribuiu para reduzir a evapotranspiração nesta parte do campo, e uma acentuada advecção local de ar seco sobre a segunda metade do campo experimental, que foi responsável pelo aumento expressivo de ET nesta área.

\section{REFERÊNCIAS BIBLIOGRÁFICAS}

BRAKKE, T.W.; VERMA, S.B.; ROSENBERG, N.J. Local and regional components of sensible heat advection. Journal of Applied Meteorology, Boston, v.17, p.955-963, 1978.

CHIN CHOY, E.W.; KANEMASU, E.T. Energy balance comparisons of wide and narrow row spacings in sorghum. Agronomy Journal, Madison, v.66, p.98-100, 1974.

HANKS, R.J.; ALLEN, L.H.; GARDNER H.R. Advection and evapotranspiration of wide-row sorghum in the Central Great Plains. Agronomy Journal, Madison, v.63, p.520-527, 1971.

LEITÃO, M.M.V.B.R. Balanço de radiação e energia numa cultura de soja irrigada. Campina Grande, PB: UFPB, 1989. 110p. Dissertação Mestrado. DCA/CMM - TD No 03

LEITÃO, M.M.V.B.R. Balanço de radiação em três ecossistemas da Floresta Amazônica: Campina, Campinarana e Mata Desa. São José dos Campos, SP: INPE, 1994. 135p. Tese Doutorado. INPE 5587-TDI.

LEITÃO, M.M.V.B.R.; AZEVEDO, P.V.; MACIEL, G.F. Balanço de radiação e energia numa cultura de soja irrigada, nas condições semi-áridas do Nordeste do Brasil. VI CONGRESSO BRASILEIRO DE METEOROLOGIA, 1990, Salvador - Ba. Anais... Salvador: Sociedade Brasileira de Meteorologia, v.1, 1990. p.27-32.

LINACRE, E.T. Estimating the net radiation flux. Agricutural Meteorology, Amsterdam, v.5, p.49-63, 1968.

MONTENY, B. Evapotranspiration de diferents couverts vegetaux in region mediterranéene semi-aride. Agricutural Meteorology, Amsterdam, v.10, p.19-38, 1972.

PRUEGER, J.H.; HIPPS, L.E.; COOPPER, D.I. Evaporation and the development of the local boundary layer over an irrigated surface in an arid region. Agricultural and Forest Meteorology, Amsterdam, v.78, p.223-237, 1996.

ROSENBERG, N.J. Frequency of potential evapotranspiration rates in Central Great Plains. Journal of Irrigation and Drainage Division of American Society Civil Engineers, New York, v.98, n.2, p.203-206, 1972.

ROSENBERG, N.J.; VERMA, S.B. Extreme evapotranspiration by irrigated alfalfa: A consequence of the 1976 midwestern drough. Journal of Applied Meteorology, Boston, v.17, p.934-941, 1978.

VERMA, S.B.; ROSENBERG, N.J.; BLAD, B.L. Turbulent exchange coefficients for sensible heat and water vapor under advective conditions. Journal of Applied Meteorology, Boston, v.11, n.1, p.330-338, 1978.

VILLA NOVA, N.A. Estudo sobre o balanço de energia em cultura de arroz. Piracicaba, SP: ESALQ-USP, 1973, 78p. Tese de Livre Docência 